\title{
CHARGE COMPENSATION AND ACCELERATION OF A THICK- WALLED HIGH-CURRENT ION BEAM IN INDUCTION LINAC
}

\author{
N.G.Belova, V.I.Karas'*, O.N.Shulika* \\ Institute of Physics \& Technology Russian Academy of Sciences, Moscow, Russia \\ * NSC KIPT, Kharkov, Ukraine
}

\section{INTRODUCTION}

At present, several methods of obtaining highcurrent ion beam, which are based on the use of induction accelerators and are applied to inertial controlled fusion (ICF) research, are being considered [1-3]. To date, kiloampere ion beams with energies of several hundred $\mathrm{keV}$ have been produced in highcurrent linear induction accelerators (linac) with collective focusing [4-6]. The power of the hollow highcurrent ion beam (HHCIB) for ICF purposes must be several orders greater, with rather stringent requirements on beam brightness. Therefore, when developing a driver for ICF on the basis of a highcurrent linac, it is necessary to investigate a number of important physical problems: (1) the formation of highcurrent beams in injector; (2) the provision of efficient magnetic insulation for accelerating gaps; (3) charge compensation of the ion beam in the transport channel and in the magneto-insulated accelerating gaps; (4) effective acceleration and stability of the ion beam in accelerating channel; and (5) transport, focusing, and space-time compression of HHCIBs.

In linac, the conventional way of charge and current compensation $[7,8]$ is inefficient. In $[9,10]$, a new mechanism for the neutralization of HHCIBs in axially symmetric magneto-insulated gaps was proposed. Its physical meaning is that a specially injected compensating electron beam drifts through the cusp due to self-consistent azimuthal magnetic field and an electric field caused by a small radial separation of ion and electron beams.

In [11-13], the investigation results of the acceleration, and the charge and current compensation of HHCIBs in one and two linac cusps are reported. These results have shown that both in the presence and in the absence of an accelerating electric field, the following effects take place: (1) charge and current compensation of HHCIB in the accelerating gaps; (2) stability of the ion beam during times that substantially exceed the inverse ion Langmuir and Larmor frequencies. The performed numerical simulations have also shown that in the drift space between two accelerating gaps the current and charge compensation of the ion beam proved to be insufficient because of a substantial difference in the electron and ion velocities acquired up to the time of the transit of the beams through the drift gap. As a result, the positive potential of the self-consistent field in the drift space leads to the spread and deceleration of the ion beam and, consequently, to the degradation of the beam brightness. The positive space charge in the drift gap can be compensated by injecting thermal electrons into it. It was shown that a preliminary injection of cold electrons permits to eliminate broadening and decelerating an ion beam in the drift gap and to provide its additional focusing.
In this work, the investigation directed to: (1) reaching of the optimal relation between the external electric field parameters and the compensating electron beam parameters; (2) defining of the optimal thickness of annular beam at which the ion beam is effectively accelerated and at a time is remaining compensated and not losing a stability.

MATHEMATICAL AND DISCRETE MODEL

To describe the collisionless plasma dynamics of beams the set of relativistic Vlasov's equations for the distribution functions of particles $f_{s}(P, R, t)$ ) in the axisymmetric $(\partial / \partial \theta=0)$ cylindrical geometry $R=(r, z)$ has been used for the investigation of the transient and stationary processes in linac (here $P$ momentum). The self-consistent electric $E(r, z)$ and magnetic $B(r, z)$ fields including in Vlasov's equations are determined by the Maxwell's equations, the right hands of which are defined as the zeroth and first moments of the distribution functions. From the set of Vlasov's equations can be obtained the set of the dynamic equations for the particles. The Maxwell's equations using the Lorentz gauge $\left(\operatorname{div} A+c^{-1} \partial \Phi / \partial t=0\right)$ can be reduced to the wave equations for the scalar $\Phi(r, z)$ and vector $A(r, z)$ potentials.

The initial velocity of a given sort $(s)$ of particles is defined by the boundary conditions for the distribution functions at $z=0$ : $f_{s}\left(m_{s} u, R, t\right)=\delta\left(u_{r}\right) \times \delta\left(u_{z}-u_{0 s}\right) \delta\left(u_{\theta}\right) \quad$ for $r_{\min } \leq r \leq r_{\max }$ and $u_{z}>0$. Here $r_{\min }$ and $r_{\max }$ are the minimum and maximum beams radii respectively which define the initial $r$-coordinates of particles, $u_{0 s}=v_{s} / \sqrt{1-v_{s}^{2}}, \quad v_{s}$ is a beam velocity. At ( $r=0, r=r_{L}$ ) the reflection regime is set. The particles exit free from the simulation region at $z=z_{L}$. At the initial time the particles are absent in modeling region.

The set of equations and the initial and boundary conditions for the potentials and distribution functions are described in detail in [15].

The configuration of the external magnetic field is defined by the expression $A_{\theta}=-B_{0} / k I_{1}(k r) \times$ $\mathrm{x} \cos (k z)$, where $I_{1}(k r)$ is the first order modified Bessel function, $B_{0}$ is the amplitude of magnetic field, and $k=K \pi / z_{L}, K$ is total number of cusps.

The discrete model, which is developed for the 
study of both relativistic and nonrelativistic axisymmetric plasma configuration, is realized as 2.5dimensional axisymmetric numerical code. The calculations were carried out using Pentium-133.

Hollow magnetized relativistic electron beam (Larmor radius $r_{L e}$ is substantially smaller than the size of the cusp $L_{z}$ and the chamber radius $r_{L}$ ) and highcurrent unmagnetized ion beam (Larmor radius $r_{L i}$ is more greater $L_{z}$ and $r_{L}$ ) were injected along $z$-axis. In all cases the beam current densities were equal to $q_{e} n_{0 e} V_{e}=q_{i} n_{0 i} V_{i}$. The ion beam velocity was $V_{i}=0.285$. The minimum and maximum beams radii were $r_{\min }=30$ and $r_{\max }=37.5$. The size of one cusp and radius of the chamber were $L_{z}=78.5$ and $r_{L}=157.5$ respectively. The amplitude of the external field was $B_{0}=1.76$. The mass ratio was $m_{i} / m_{e}=100, m_{e}=20 m_{0}$. The number of particles in the cell was $N_{e}=64$, $N_{i}=180$. The number of the nodes was equal to $\left(J_{z} x\right.$ $\left.J_{r}\right)=(64 \times 64)$. The time step was varied within the range $0.025-0.05 \omega_{p e}^{-1}$ for solving the equations of motion and $0.0125-0.025 \omega_{p e}^{-1}$ when solving the wave equations. The parameters of the electron and ion beams have satisfied the condition of the electron beam transport together the ion beam through the magnetoinsulated accelerating gap $\left(v_{e}=0.8 \div 0.85\right)$. The preliminary injection of the thermal electrons with the Maxwellian distribution function with the temperature $T_{c e}=0.002$ into drift gap was used.

\section{RESULTS OF SIMULATION}

Further (below) the results of three cases distinguished by the potential difference across one cusp are discussed. The distributions presented in Fig. 1 show that the applied external electric field, which accelerates ions and retards electrons, does not prevent the electron beam drift through the accelerating gaps. It is clearly seen that not only the charge but also the current compensation of the ion beam occur. The ion beam generally retains monoenergetic shape, because its spread in $v_{z}$ and $v_{r}$ does not exceed $10 \%$.

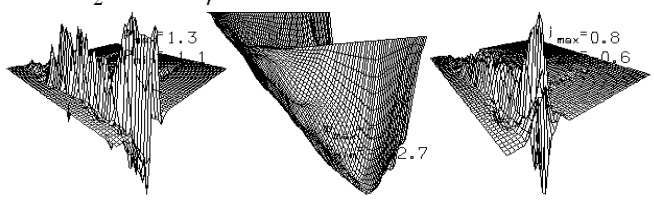

Fig. 1. Distribution of the total charge density $\rho(r, z)$, the scalar potential $\Phi(r, z)$ and the total axial current densities $j_{z}(r, z)$ at $t=440 \omega_{\text {pe }}^{-1}$ for the case with additional electron injection into drift gap and thickwall ion beam.

More optimal relation between the potential difference $\Delta \Phi=15.0$ and the electron beam energy was at the parameters $v_{e}=0.85, \gamma \approx 1.9$. The kinetic energy of electron beam $\varepsilon_{e b} \approx 18.0$ was sufficient to overcome the potential difference. As a results the ion beam is accelerated and then its velocity is a constant in all time of simulation. Fig. 1 illustrates that the selfconsistent field of the positive charge is suppressed, and an additional focusing of ion beam by the negative space charge of the thermal electrons occur also. As a result the better characteristics of the ion beam can be produced in linac.

The preliminary injection of the thermal electrons into the drift gap upgrades the characteristics of HHCIB. At Fig. 2 the time dependences of the mean ion beam velocities for three cases are presented.

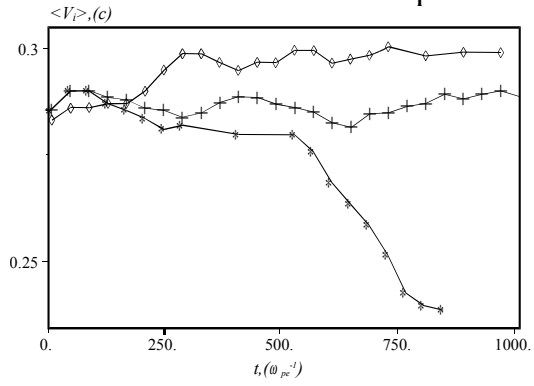

Fig. 2. Distribution of the mean ion velocity $\left\langle V_{i}(t)\right\rangle$ : (*) - without thermal injection into accelerating gap;

$(+)$ - with injection and without accelerating field;

$(\diamond)$ - with injection and accelerating field.

\section{CONCLUSION}

Results of the 2.5-dimensional numerical simulation of HHCIB dynamics in two magnet-isolated accelerating gaps separated by the drift gap are presented. With the aim of increasing the ion beam acceleration rate in the two cusps linac we have studied the optimal correlation between an accelerating electric field and an electron beam energy. We obtained the restriction on the wall thickness of annular HHCIB which ensure the following: (1) charge- and current compensation is homogeneous on the ion beam crosssection; (2) a penetration depth of the accelerating electric field is considerably greater than the beam wall thickness.

This work was partly suppoted by State Foundation for Fundamental Researches (grant $1 / 2.52 / 38)$.

\section{REFERENCES}

1. A.I. Fattens, and D. Keeffe, Proc. Lin. Accel. Conf., Santa Fe, LA9234c, 205 (1981).

2. R.O. Bangerter, Part. Accel. 37-38, 3 (1992).

3. S.A. Slutz, P. Primm, T. Renk et.al. Final Report, SAND97-0344 UC-414, March 1997

4. V.A. Kiyashko, Yu.E. Kolyada, E.A. Kornilov, Ya.B. Fainberg, Pis'ma Zh. Tekhn. Fiz. 3, 1257 (1977)

5. S.Jr. Humphries, Nucl. Fusion 20, 1549 (1980).

6. I.S. Roth, J.D. Ivers, J.A. Nation Proc. VI Int. conf. on high power particle beams, Kobe, 1986.

7. W. Peter, N. Rostoker, Phys. Fluids 25, 730 (1982);

8. W. Peter, A. Ron, N. Rostoker, Phys. Fluids 22, 1471 (1979).

9. V.I. Karas', V.V. Mukhin, V.E. Novikov, A.M. Naboka, Fiz. Plazmy 13, 494 (1987).

10. V.I. Karas', V.A. Kiyashko, E.A. Kornilov, Ya.B. Fainberg, Nuclear Instruments and Methods in Physics Research A278, 245 (1989).

11. N.G. Belova, V.I. Karas', Yu.S. Sigov, Fiz. Plazmy 16, 209 (1990).

12. N.G. Belova, V.I. Karas', Part. Accel. 37-38, 225 (1992).

13. N.G. Belova, V.I. Karas', Proc.IEEE Part.Accel. Conf. (Washington DC, V.I, 1993).

14. V.I. Karas', N.G. Belova, Proc. X Int. Conf. on High Power Particle Beams BEAMS'94 (San Diego, 1994).

15. N.G. Belova, V.I. Karas', Plasma Physics Reports 21, 1005 (1995). 16. N.G. Belova, V.I. Karas', Plasma Physics Reports 23, 328 (1997). 